\title{
Partial Discharge On-line Monitoring for HV Cable Systems Using Electrooptic Modulators
}

\author{
Y. Tian, P. L. Lewin \\ High Voltage Laboratory \\ Department of Electronics and Computer Science, University of Southampton \\ Southampton, Hampshire SO17 1BJ, UK \\ D. Pommerenke \\ University of Missouri-Rolla \\ EMC Group \\ Rolla, MO, USA \\ J. S. Wilkinson \\ Optoelectronics Research Centre \\ University of Southampton \\ Southampton, Hampshire SO17 1BJ, UK \\ and S. J. Sutton \\ National Grid Transco \\ Warwickshire, UK
}

\begin{abstract}
A technique for the remote inspection and monitoring of partial discharge (PD) activity using an optical network is described. The network uses a $\mathrm{LiNbO}_{3}$ modulator to modulate the intensity of the transmitted laser light approximately proportional to the voltage applied across the modulator. The laser light is transmitted along an optical fibre and measured remotely by a high-speed optical receiver. A capacitive coupler has been used to detect partial discharge activity and act as the modulator's input voltage source. The electro-optic modulator is passive and does not require a power supply at the site of the PD detection sensor. The system has the additional advantages of being immune to electromagnetic interference, having very little signal transmission attenuation, with good sensitivity, compact size, as well as being convenient to use and safe. Both simulation experiments and practical PD tests on two cable systems have indicated that the optical system provides a feasible remote PD monitoring technique for high voltage cable systems.
\end{abstract}

Index Terms - Partial discharge, $\mathrm{LiNbO}_{3}$ electro-optic modulator, capacitive coupler, on-line monitoring, high voltage cable.

\section{INTRODUCTION}

$\mathrm{P}$ ARTIAL discharge (PD) activity can occur in XLPE cables and this can lead to insulation breakdown and may ultimately lead to cable failure. Therefore, PD detection is an important condition monitoring tool for cable systems. Compared with cables that are manufactured under controlled factory conditions, cable joints are often installed on-site in less favorable conditions. Defects that generate PD are more likely to exist within cable joints than in the cable itself.

Manuscript received on 30 January 2003, in final form 2 December 2003.
Conventional electrical PD testing has been used for many years to evaluate cable quality. Such tests are generally performed off-line within a well-screened laboratory using internationally agreed standard methods [1]. This approach should be able to detect the permissible discharge quantity regulated for the test object. However, in many situations the purpose of PD measurement is to detect any deterioration of the apparatus during its normal use, through on-line monitoring. For on-line monitoring, when the deterioration reaches a certain level, warnings should be given in order to prevent the occurrence of breakdown. Apart from faults in cables and cable accessories due to long-term aging effects, spontaneous faults 
or breakdown can occur. It is important that on-line monitoring is also able to detect newly arising defects. Several on-line PD monitoring methods for cable systems, including the acoustic emission sensors, inductive high frequency current transducers, and very high frequency capacitive couplers, have been investigated extensively by the authors [2]. In particular, capacitive couplers have been demonstrated to be effective PD on-line detection sensors [3].

PD signals detected by capacitive couplers, which are usually installed close to the cable joints, need to be transmitted to the measuring equipment for data acquisition, processing and further analysis. The simplest approach for PD signal transmission is via electrical coaxial cables. However, there are disadvantages with this means of signal transmission. A cable tunnel may be several kilometers long and have many cable joints, for PD on-line monitoring, the measuring equipment must be placed outside the cable tunnel. When partial discharges occur in a distant cable joint, the discharge signals detected by the capacitive couplers may have to travel a significant distance before measurement. This could result in signal attenuation and consequently decrease the PD detection sensitivity. In addition, electrical interference can be severe under normal operating conditions, and noise will be coupled into the long sensor lead to be captured by the measuring equipment. This will further decrease the detection sensitivity and in some situations noise may totally bury the real PD signal.

A method to overcome signal transmission attenuation and electromagnetic interference is to use an optical fibre system for signal transmission [4-6]. Generally discharge signals detected by the sensors are fed into an optical transmitter, where they are converted into optical signals. The optical signals are then transmitted via an optical fibre, and then converted back to electrical signals using an optical receiver before being captured by the measuring equipment. PD signal transmission via optical fibre shows reduced signal transmission attenuation compared with electrical transmission, and is immune from electromagnetic interference. The optical fibre also provides electrical isolation of the measuring equipment. The major disadvantage for this method is that the optical transmitter requires a power supply to operate and this is not very convenient, especially for on-site applications.

Many optical modulation techniques are based on the analysis of light polarization that is altered after light has passed through a dielectric material. Due to one of the electro-optic effects or the photo-elastic effect, the electric field or the mechanical stress inside a dielectric material modifies the refractive index of the material and thus produces an additional optical phase difference [7]. The Pockels electro-optic effect has been used to investigate surface discharge phenomena [8]. Optical interferometry technique has been used to monitor PDs in power trans- formers by means of ultrasonic transducers [9-11]. In this case the ultrasonic pressure waves due to partial discharge activities were used to phase modulate the optical beam propagating through a fibre which is later received using photodetectors.

This paper investigates the feasibility of a PD on-line monitoring system involving $\mathrm{LiNbO}_{3}$ electro-optic (EO) modulators. The technique uses an $\mathrm{EO}$ modulator to convert the electrical signal of the partial discharge sensor into an optical signal [12-14]. The optical signal is powered and transmitted along an optical fibre using a remote fibre laser and detected by an optical receiver. The EO modulator is compact, sensitive, reliable and passive requiring no electric power. Overall the use of the optical measurement system has the following advantages: it is immune to electromagnetic interference; there is very little signal transmission attenuation; it has high detection sensitivity; it is passive without low voltage power supply requirement at the cable joint; it can be buried underground; it provides electrical isolation and protection to both personnel and measuring equipments; it is compact and convenient to use.

\section{ELECTRO-OPTIC EFFECT AND MEASUREMENT SYSTEM}

The fundamental principle of the electro-optic effect is that an applied field affects an electro-optic crystal by changing its indices of refraction. Consider the case of a Lithium Niobate crystal $\left(\mathrm{LiNbO}_{3}\right)$ where a voltage $\mathrm{V}$ is applied on its side plates, as shown in Figure 1. The light propagating in the $\mathrm{z}$ direction through the $\mathrm{LiNbO}_{3}$ crystal can be resolved into two components in the $\mathrm{x}^{\prime}$ and $\mathrm{y}^{\prime}$ directions that have a phase difference or phase retardation of $[15,16]$

$$
\Delta \phi=\frac{2 \pi}{\lambda} n^{3} r V
$$

where $\lambda$ is the wavelength of the incident light, $n$ is the index of refraction, $r$ is an electrooptic coefficient of the crystal, and $V$ is the applied voltage.

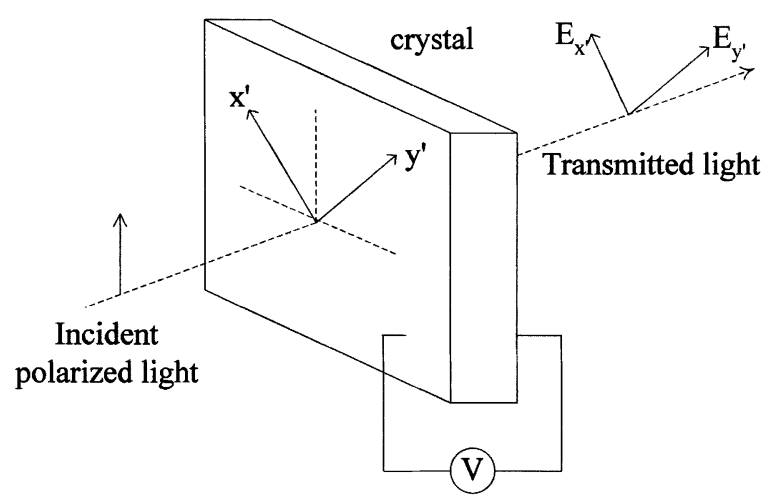

Figure 1. $\mathrm{A} \mathrm{LiNbO}_{3}$ crystal as an electrooptic modulator. 


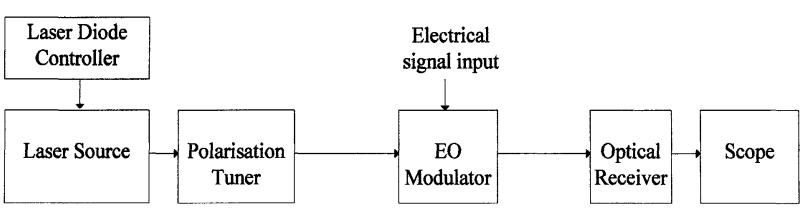

Figure 2. The measurement system.

Equation (1) indicates that the phase shift $\Delta \phi$ depends linearly on the applied voltage $V$. In the case of a modulator which uses polarization rotation, a polarization analyzer is placed after the crystal and the optical amplitude transmitted through the analyzer is a cosinusiodal function of the phase shift. Thus the variation of the applied voltage can vary the phase shift and consequently vary the transmitted optical power from the crystal. This is similar to the operating principal of the $\mathrm{LiNbO}_{3}$ waveguide modulator used in the proposed measurement system, where modulation is achieved by splitting the light into two separate paths, modulating the phase of one, and recombining to result in an interferometric signal which is similarly a cosinusoidal function of $\Delta \phi$.

The diagram of the optical measurement system is shown in Figure 2. The measurement mechanism uses an electrical signal that relates to local partial discharge activity within the cable system and applies it across an optical fibre coupled $\mathrm{LiNbO}_{3}$ waveguide modulator, which modulates the intensity of the transmitted laser light as an approximately linear function of the voltage applied across it, for sufficiently small values of $\Delta \phi$, when suitably biased. The optical network supplies polarized laser light at one end of a fibre optic cable, connected to the remote $\mathrm{LiNbO}_{3}$ modulator, and monitors the optical signal received at the other end of the fibre. The $\mathrm{LiNbO}_{3}$ modulator requires no power source for it to operate. Using optical fibre as the transmission medium allows the remote monitoring of a particular location and enables devices such as the laser source and receiver to be located wherever convenient. Potentially, new cables could be laid together with optical fibres and new joints could be designed to include optical network ready PD sensors.

The laser source used in this investigation was a C15 KOH1001 erbium-doped fibre laser (Koheras A/S, Denmark) having a centre wavelength of $1550 \mathrm{~nm}$, maximal power of $9.3 \mathrm{~mW}$, and a single transverse mode output. The fibre laser was pumped with a $975 \mathrm{~nm}$ laser diode with a current of $50-180 \mathrm{~mA}$, driven by a laser diode controller type ITC 510 (Profile GmbH, Germany) having a temperature control range of $-50-140{ }^{\circ} \mathrm{C}$ and laser current range of $0 \pm 1 \mathrm{~A}$.

A polarisation controller was placed at the output of the laser to ensure that the input light for the modulator was linearly polarized. The in-line polarization controller model PC1100-15-F/A (Fiberpro Company, USA) has an inline attenuation of $0.40 \mathrm{~dB}$ and polarization extinction ratio of $35.06 \mathrm{~dB}$ at $1570 \mathrm{~nm}$.

The $2.5 \mathrm{~Gb} / \mathrm{s} \mathrm{LiNbO}_{3}$ modulator (Aeroflex-Trilink Corp., USA) has an extinction ratio of $22 \mathrm{~dB}$, an insertion loss of $4.6 \mathrm{~dB}$ and maximal RF input voltage of $2.6 \mathrm{~V}$ at $1550 \mathrm{~nm}$ wavelength and $25^{\circ} \mathrm{C}$. The modulator has dimensions of $80 \times 10 \times 10 \mathrm{~mm}^{3}$.

A high-speed low noise optical receiver model 1611-FCAC (New Focus Inc., USA) was used to detect the optical signal. This device has a wavelength range of $900-1700$ $\mathrm{nm}$, maximum output current of $10 \mathrm{~mA}$ (into $50 \Omega$ ), response of $1.005 \mathrm{~A} / \mathrm{W}$ at $1550 \mathrm{~nm}$, and frequency range between $30 \mathrm{kHz}$ and $1 \mathrm{GHz}$. Use of a power supply for this component is not an issue, since this device would most likely be located at a ground station and not at a remote field location, unlike the EO modulator.

The output of the optical receiver was monitored using a Tektronix ${ }^{\circledR}$ digital oscilloscope with a maximal sampling rate of $1 \mathrm{GS} / \mathrm{s}$ and bandwidth of $500 \mathrm{MHz}$.

\section{RESULTS \\ 3.1 FEASIBILITY TESTS}

The performance of the optical measuring system was initially investigated using a pulse generator to provide a signal input into the EO modulator. The experimental arrangement is as shown in Figure 2. The pulse generator provides an output pulse as the EO modulator input, and a synchronized pulse to one channel of the oscilloscope to provide the electrical pulse reference.

Figure 3 shows the pulse generator output pulse and the optical receiver output pulse. The two pulses were captured simultaneously with the pulse generator pulse acting as the oscilloscope triggering source. In this case the laser controller current is $70 \mathrm{~mA}$. The time delay between the two pulses is caused by the signal transmission delay between the EO modulator and the oscilloscope including the optical fiber between the modulator and the
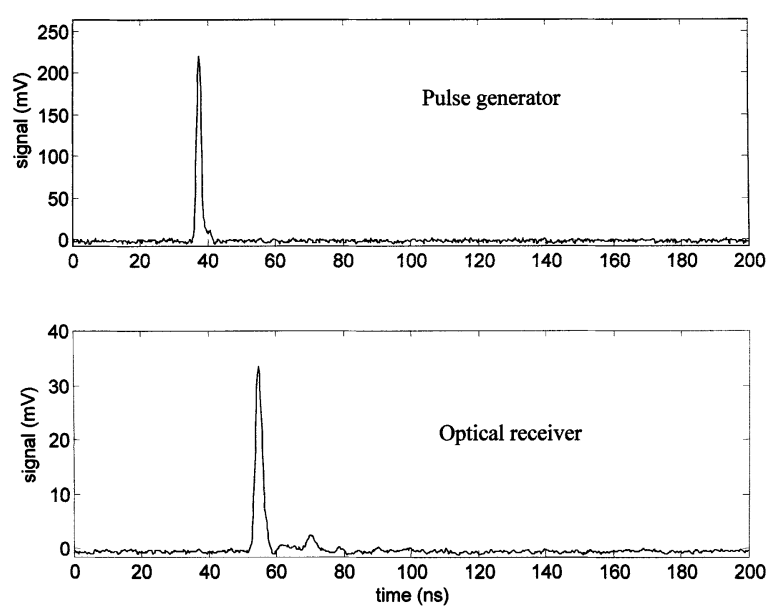

Figure 3. Pulse generator output and optical receiver output pulses. 


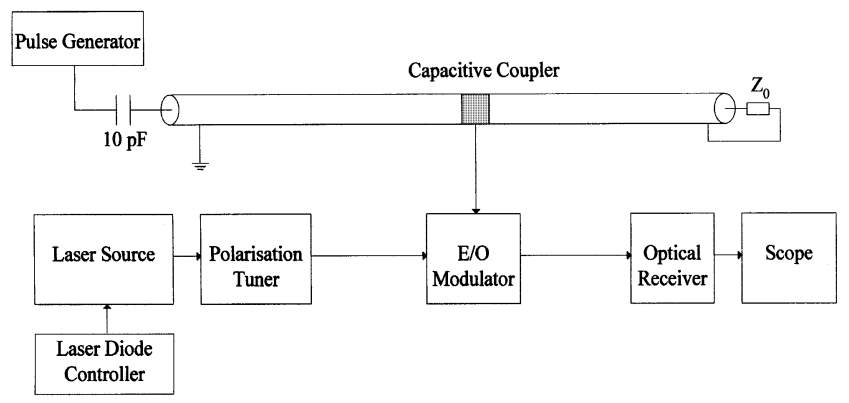

Figure 4. Optical measurement system with capacitive coupler and PD simulator.

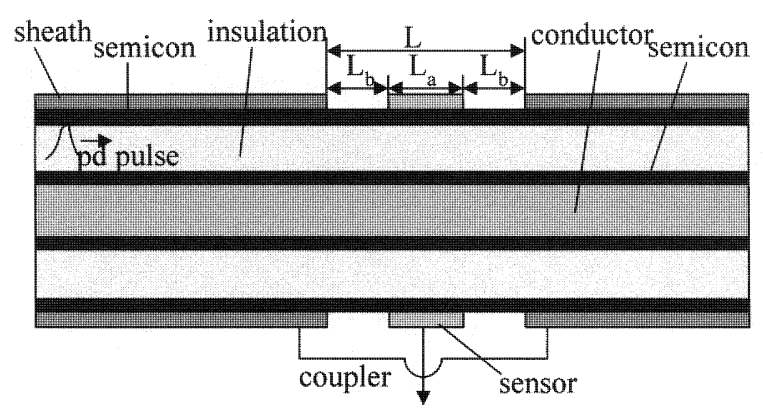

Figure 5. Simple implementation of a capacitive coupler for use in laboratory measurements

optical receiver, and the electrical BNC cable between the optical receiver and the scope. The optical receiver signal magnitude can be increased by increasing the laser controller current and tuning the polarization controller to obtain the optimally polarized light for the EO modulator.

To investigate the response of the optical measurement system to typical discharge signals that can occur within cables, a simulation experiment has been undertaken (Figure 4). In this case capacitive coupler output signals were used as the electrical modulation inputs for the EO modulator. A simple capacitive coupler was installed on a $3 \mathrm{~m}$ cross-linked polyethylene (XLPE) cable section, and its configuration is shown in Figure 5. The cable outer metal sheath of length $\mathrm{L}$ ( $100 \mathrm{~mm}$ in this case) has been removed, and tin tape was wrapped around the cable outer semicon layer over a distance $\mathrm{L}_{\mathrm{a}}(40 \mathrm{~mm}) . \mathrm{L}_{\mathrm{b}}(30 \mathrm{~mm})$ is the distance between the tape and the cable sheath. The higher potential electrode of the coupler is attached on top of the semicon screen that serves to provide a smooth interface between the dielectric and the cable metallic ground screen and remove the electrical stress concentration. The lower potential electrode of the coupler is defined by the metallic ground screen. The conductivity of the semicon layer has frequency dependent characteristics. The sensitivity of capacitive couplers in PD measurement has been demonstrated as partial discharges less than $3 \mathrm{pC}$ can be clearly detected by capacitive couplers installed $1.5 \mathrm{~m}$ away from the PD location $[2,3]$.
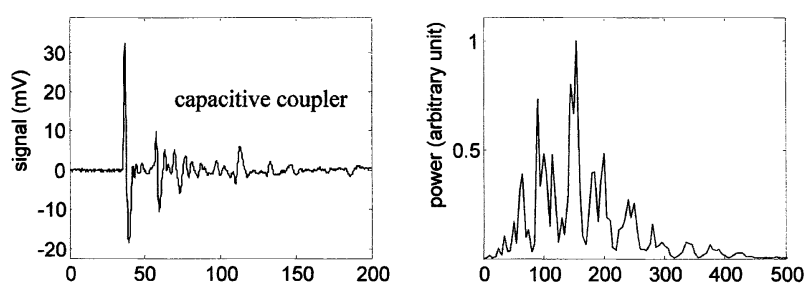

(a)

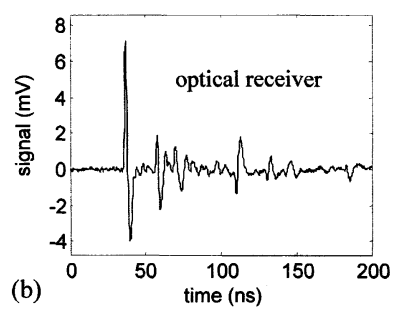

(c)

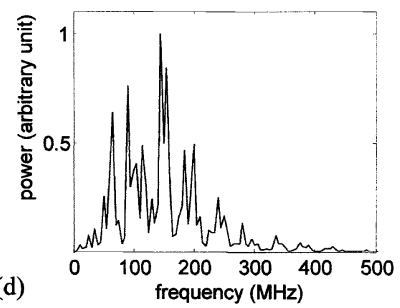

Figure 6. Capacitive coupler and optical receiver outputs when an equivalent charge of $10 \mathrm{pC}$ was injected into a XLPE cable section. a, capacitive coupler; b, optical receiver; $c$, spectrum of the capacitive coupler signal; d, spectrum of the optical receiver signal.

A step wave from the pulse generator was injected into one cable end via a $10 \mathrm{pF}$ capacitor. In theory the equivalent discharge quantity $(q)$ can be considered as

$$
q=C \cdot V
$$

where $C$ is the capacitor capacitance and $V$ is the magnitude of the step wave.

Figure 6 shows the capacitive coupler output signal, the optical receiver output signal, and their relevant power spectra, when a step wave of $1 \mathrm{~V}$ was injected into the cable via the $10 \mathrm{pF}$ capacitor. The laser controller current is $80 \mathrm{~mA}$. In this case the two signals were obtained separately with the pulse itself as the triggering source for the oscilloscope. The power spectra were normalized to the relevant maximal magnitude. The power spectra indicated that similar frequency characteristics were obtained for the capacitive coupler and the optical receiver signals. The EO modulator has a bandwidth of $2.5 \mathrm{GHz}$ and the optical receiver has a frequency bandwidth up to $1 \mathrm{GHz}$. Consequently the frequency response of the optical measurement system is determined by the capacitive coupler, which in general operates in the very high frequency range of approximately three hundred $\mathrm{MHz}$.

The influence of the laser controller current on the optical measuring system performance has been investigated. At first the relationship between the laser controller current and the laser source output power was studied, as shown in Figure 7. The laser power was measured using an optical power meter. The result indicates that once the laser current surpasses a threshold level, the optical power increases almost linearly with the controlling current. The effect of the laser controller current on the optical receiver signal magnitude was then investigated. In this case a step wave of $1 \mathrm{~V}$ was injected into the 


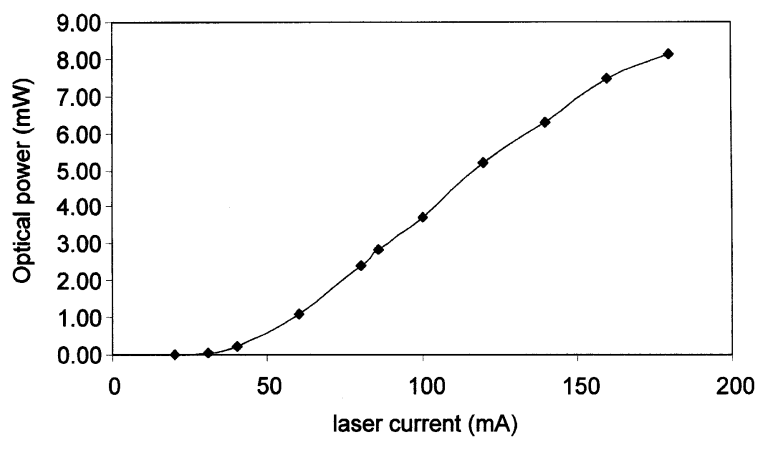

Figure 7. Laser output power vs. laser controller current.

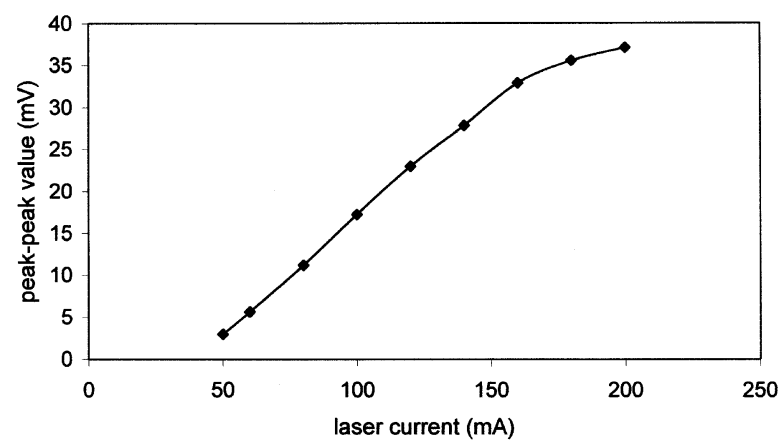

Figure 8. Optical receiver signal peak-to-peak value vs. laser controller current.

cable via the $10 \mathrm{pF}$ capacitor, and the capacitive coupler signal was injected into the EO modulator. Figure 8 indicates that the peak-to-peak value of the optical receiver signal increases with the increase of the laser current within the current range of $50-200 \mathrm{~mA}$, which is in good agreement with Figure 7. Larger laser controller current generates higher laser output power, which in turn leads to higher optical receiver signals.

Pulse generator outputs with various magnitudes have also been applied. The obtained capacitive coupler and optical receiver signals have similar waveforms but with different magnitudes which relates to the injected magnitudes. This experiment demonstrates the feasibility of using the optical measurement system to measure discharge signals via a capacitive coupler.

\subsection{MEASUREMENTS OF PD ACTIVITY WITHIN A 90 kV HV CABLE AND A 132 kV JOINT}

Having established that the proposed method is feasible, the system has been applied to detect practical partial discharges produced within a $90 \mathrm{kV}$ XLPE cable section having de-ionized water terminations. The test arrangement is shown in Figure 9. To provide the discharge apparent quantity, PD conventional electrical detection according to IEC-60270 has also been applied. The partial discharge detector is a Robinson ${ }^{\circledR}$ model 5 type 700 . In

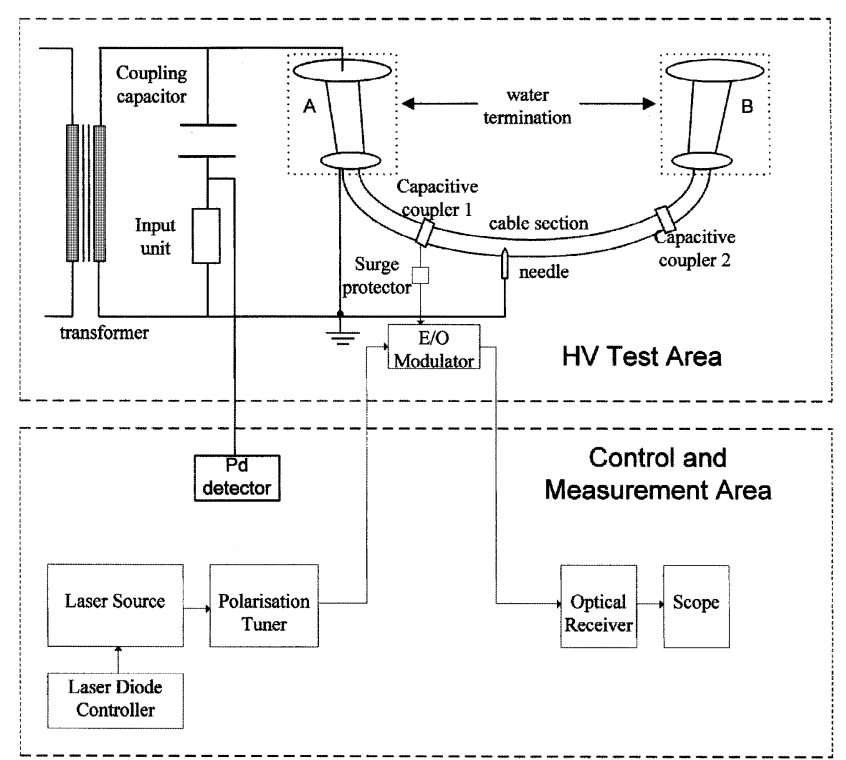

Figure 9. Application of the optical measurement system to detect PDs produced from a XLPE cable loop with de-ionized water terminations.

order to produce partial discharges from the cable section, a sharp tungsten needle was inserted into the cable insulation, approximately $3 \mathrm{~mm}$ away from the inner conductor semicon screen. The tungsten needle has a $1 \mathrm{~mm}$ diameter shank leading to $3 \mu \mathrm{m}$ pin-tip radius. In this case the construction of the capacitive coupler was more complicated than that shown in Figure 5. A polyethylene (PE) film was placed over the semicon layer and the sensor was mounted on the PE film. A $50 \Omega$ coaxial lead was connected to the sensor and its earth to the sheath of the cable. Bubble wrap was placed around the sensor and semicon and the whole assembly covered in aluminium foil to reduce electrical noise. The foil ensures that the cable sheath conductor is continuous. A surge protector with a bandwidth of $1000 \mathrm{MHz}$ was placed between the capacitive coupler and EO modulator. The surge protector was used to protect the EO modulator from any possible over-voltage or breakdown, even though the EO modulator itself has a passive structure. In addition, the optical fibres between the HV cable system and the measuring equipment provide electrical isolation, thus preventing any damage to the measuring equipment should the HV cable breakdown.

Figure 10a shows the PD signal measured on the capacitive coupler and its respective optical receiver output signal, at a test voltage of $6.2 \mathrm{kV}$. The conventional PD detector reveals that the discharge level is about $10-20 \mathrm{pC}$; Figure 10b shows the PD capacitive coupler signal and its relevant optical receiver output signal, at a test voltage of $6.5 \mathrm{kV}$. The conventional PD detector reveals that the discharge level is about $30-40 \mathrm{pC}$ for the latter case. In both cases the laser controller current is $160 \mathrm{~mA}$. It can 


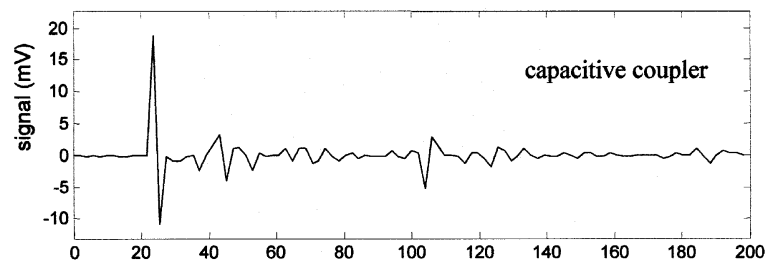

(a)
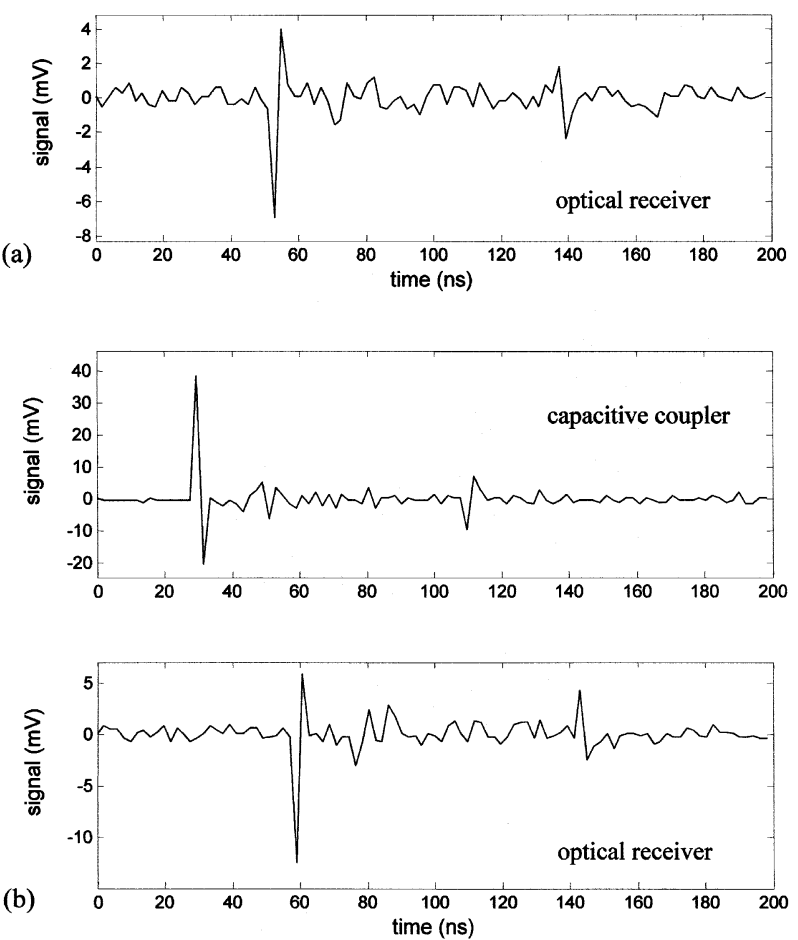

Figure 10. PD signals detected by the capacitive coupler and the optical receiver. a, test voltage of $6.2 \mathrm{kV}$; b, test voltage of $6.5 \mathrm{kV}$.

be observed in Figure 10 that the polarity of the received optical signal in this measurement is reversed with respect to that in Figure 3. This is because the operating point of the optical modulator was adjusted between these measurements.

Figure 11 shows the partial discharge signal measured by the optical receiver and the PD conventional detector respectively. The $50 \mathrm{pC}$ calibration pulse from the PD conventional detector is also provided. The results obtained indicate that in this case the discharge quantity is around $15 \mathrm{pC}$ and the relevant optical receiver signal magnitude is around $7 \mathrm{mV}$. The background noise level for the optical receiver signal is around $1 \mathrm{mV}$. Given that for reliable partial discharge measurement a minimum SNR of two is required, the measurement sensitivity of the optical measuring system for this case can be estimated as around $4 \mathrm{pC}$. It should be noted that the optical receiver has a bandwidth of $1 \mathrm{GHz}$ but the sampling rate is only $500 \mathrm{MS} / \mathrm{s}$ (with 50,000 points on a $100 \mu \mathrm{s}$ time scale). Therefore, the PD signal components above 250
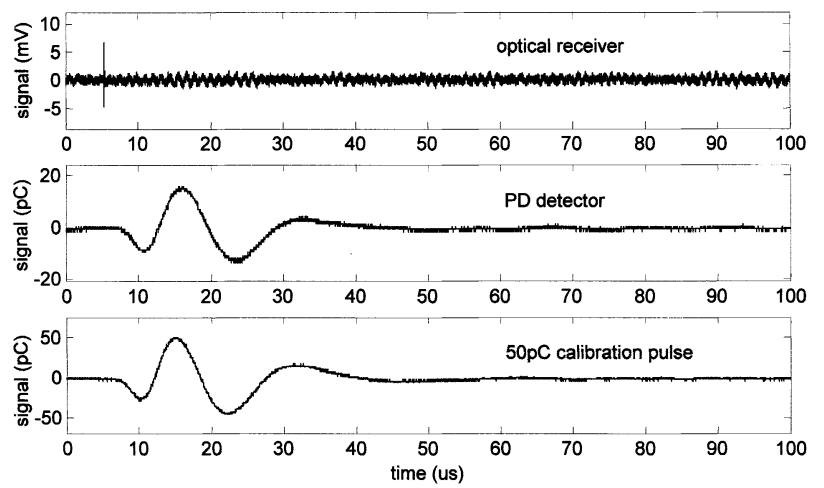

Figure 11. PD signals detected by the optical receiver and conventional PD detector.

$\mathrm{MHz}$ have not been detected. The measurement sensitivity of the optical measuring system could be improved by using higher sampling rates, but this implies the need for greater data storage space. As shown in Figure 11 the PD signal is presented as a spike from the optical receiver, but as an extended waveform from the PD conventional detector, which operates in the frequency range up to only several hundred $\mathrm{kHz}$.

The optical measurement system has also been applied to detect partial discharges produced from a $132 \mathrm{kV}$ cable/joint loop. The test arrangement is shown in Figure 12. Two $132 \mathrm{kV}$ XLPE cable sections were connected by a prefabricated cable joint. The main insulation material of the cable joint is ethylene propylene rubber (EPR), whereas the cable insulation is XLPE. The cable ends were connected to oil-filled cable terminations. Conventional PD electrical detection was also applied to provide the discharge apparent quantity. A conducting paint in the shape of ' $v$ ' was installed on top of the cable XLPE be-

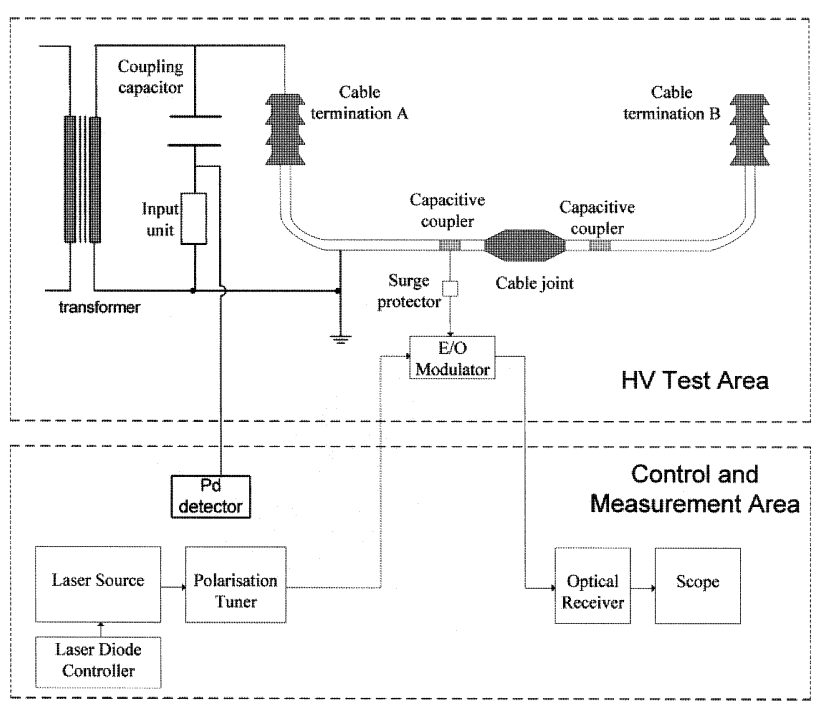

Figure 12. Application of the optical measurement system to detect PDs produced from a $132 \mathrm{kV}$ cable/joint loop. 

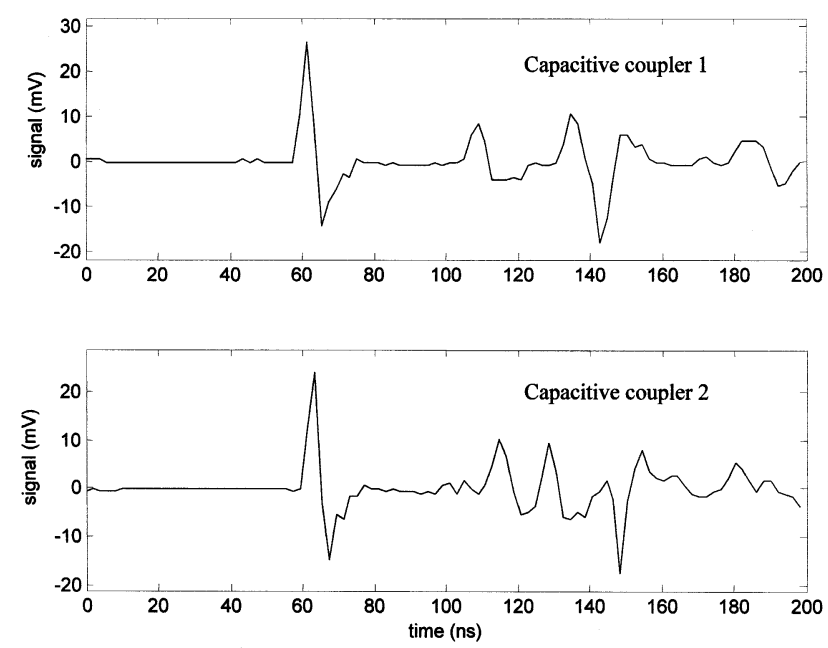

Figure 13. PD signals detected by two capacitive couplers.

tween the cable joint stress cone (conductor) and the cable joint outer semiconducting layer. This acted as the PD source within the cable joint. The capacitive couplers were installed on the cable section close to the cable joint.

Figure 13 shows the output signals from two capacitive couplers that are installed either side of the cable joint, at a test voltage of $21 \mathrm{kV}$. There are reflected pulses for both coupler outputs, and investigation indicates that these reflections occur at the two cable terminations. In this case capacitive coupler 1 leads capacitive coupler 2 with a time of flight of approximately 2 ns. This corresponds to a distance of about $0.4 \mathrm{~m}$ considering the typical discharge pulse traveling speed within the cable system. By further consideration of the distance between the two capacitive couplers, judgment can be made whether partial discharges are from within the cable joint, from the left hand side of the joint or from the right hand side of the joint, and in the first case, the approximate location of the PD site within the cable joint itself.
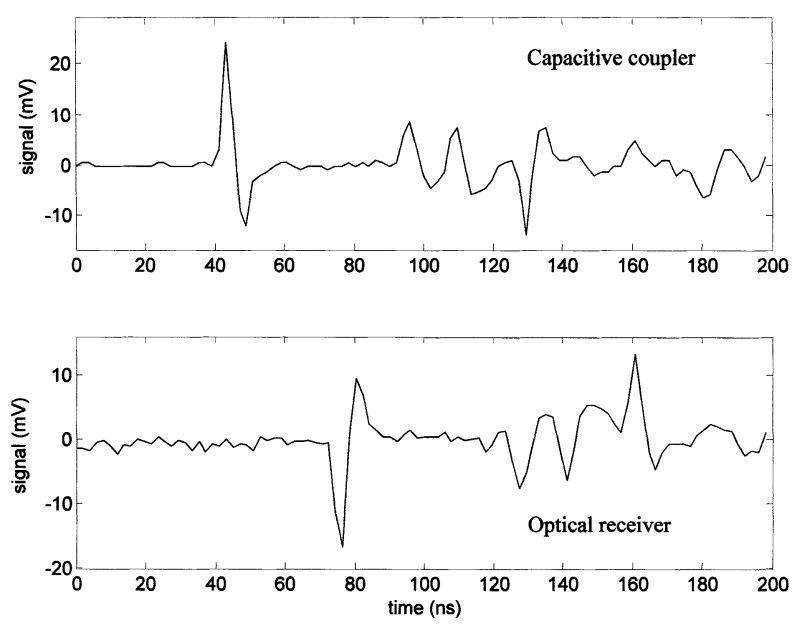

Figure 14. PD signals from the cable/joint loop as detected by the capacitive coupler and the optical receiver.
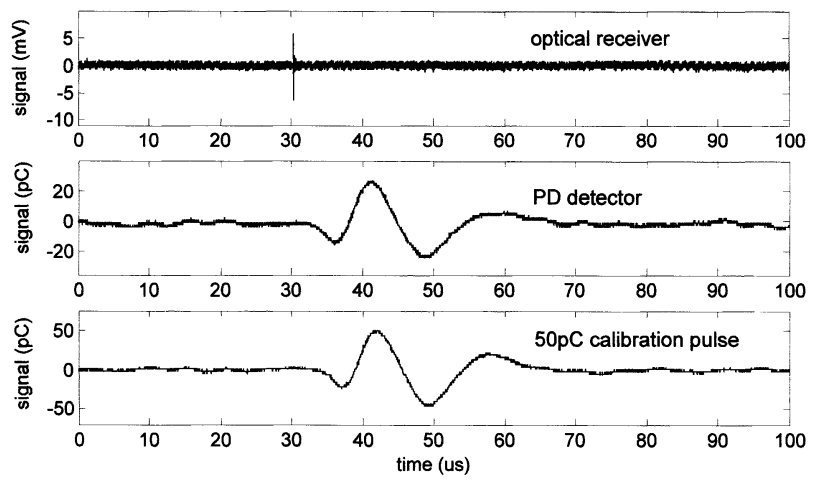

Figure 15. PD signals from the cable/joint loop measured by the optical receiver and PD conventional detector.

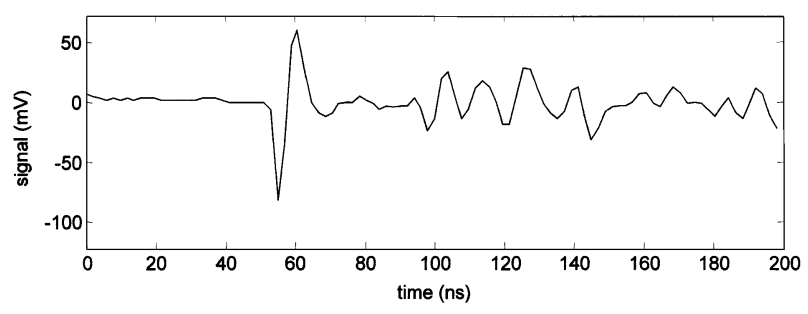

Figure 16. PD signals from the cable/joint loop detected by the optical receiver at an enhanced test voltage.

Figure 14 shows the capacitive coupler-measured PD signal and its respective optical receiver measured signal, at a test voltage of $20 \mathrm{kV}$. In this case the laser controller current is $160 \mathrm{~mA}$. The conventional PD detector reveals that the discharge level is between $20-30$ pC. Figure 15 shows a PD signal measured by the optical receiver and the PD conventional electrical detector respectively, together with the $50 \mathrm{pC}$ calibration pulse. For this particular set of data the discharge quantity is $25 \mathrm{pC}$ and the relevant optical receiver signal magnitude is around $6 \mathrm{mV}$. The background noise level for the optical receiver signal is less than $1 \mathrm{mV}$. The measurement sensitivity of the optical measuring system for this case can be estimated as approximately $8 \mathrm{pC}$. Figure 16 shows the optical receiver measured PD signal at the same laser controller current but at an enhanced test voltage of $31 \mathrm{kV}$. The PD conventional detector indicates that the PD level is around $100-120 \mathrm{pC}$. This has in turn produced larger optical receiver signals.

\section{CONCLUSIONS}

This paper has investigated the application of a PD online monitoring technique for high voltage cable systems using a $\mathrm{LiNbO}_{3}$ electrooptic modulator. Both simulation experiments and practical PD tests on a $90 \mathrm{kV}$ XLPE cable loop and a $132 \mathrm{kV}$ cable/joint loop have verified the principle and demonstrated the effectiveness of such a PD monitoring technique. This proposed technique does not require any power supply at the site of the detection sensors (in this case capacitive couplers) as the electrooptic 
modulators are passive. The detected PD signal of around a few tens of $\mathrm{mV}$ is connected to the EO modulator without any signal amplifiers. The obtained results indicate that practical partial discharges of $10-20 \mathrm{pC}$ can be clearly measured by the optical monitoring system within the laboratory, and the signal to noise ratio shows that discharges of a few pCs are also measurable using the system. Further optimization of modulator sensitivity and reduction in receiver noise are expected to improve the detection limit further. The monitoring system is sensitive, safe, compact, easy to operate, immune from electromagnetic interference, reliable and shows very little transmission attenuation. Consequently it is well suited for application to PD on-line monitoring for HV cable systems.

Further work needs to be completed before this technique may be practically applied to in-service high voltage cable systems. The optical measuring system needs to be calibrated to relate the measured signal in $\mathrm{mV}$ to the discharge apparent quantity in $\mathrm{pC}$. If such technique is to be applied to practical HV cable systems such as a threephase cable tunnel with many cable joints, problems and methodologies regarding data acquisition, transmission and processing need to be solved and established. For instance, consideration of the number of modulators needed for a complete three phase cable circuit, e.g. three modulators or just one modulator for one set of three-phase joints; the number of laser sources required; the amount of optical fibres needed; the kind of multiplexing methods to be applied etc. It would also be valuable to investigate the feasibility of implementing non-linear polymeric materials acting as optical modulators designed into the fabric of the cable joint itself.

\section{ACKNOWLEDGEMENTS}

The authors would like to acknowledge the financial support from National Grid Transco (UK), and its permission to publish this work.

\section{REFERENCES}

[1] IEC Standard 60270, High Voltage Testing: Partial Discharge Measurement, 1996.

[2] Y. Tian, P. L. Lewin, A. E. Davies, S. G. Swingler, S. J. Sutton and G. M. Hathaway, "Comparison of On-line Partial Discharge Detection Methods For HV Cable Joints", IEEE Trans. Dielectr. Electr. Insul., Vol. 9, pp. 604-615, 2002.

[3] Y. Tian, P. L. Lewin, A. E. Davies, S. J. Sutton and S. G. Swingler, "Partial Discharge Detection in Cables Using VHF Capacitive Couplers", IEEE Trans. Dielectr. Electr. Insul., Vol. 10, pp. 343-353, 2003.

[4] M. Ekberg, A. Gustafsson, M. Leijon, T. Bengtsson, T. Eriksson, C. Tornkvist, K. Johnsson and L. Ming, "Recent Results in HV Measurement Techniques", IEEE Trans. Dielectr. Electr. Insul., Vol. 2, pp. 906-914, 1995.

[5] S. Ji, D. Xu and Y. Li, "Application of optical fibre transmitting system in transformer's vibration measurement", Conf. Electr. Insul. Dielectr. Phenomena, Cancun, Mexico, pp. 910-913, 2002.

[6] D. W. Auckland, A. J. McGrail, C. D. Smith, B. R. Varlow, J. Zhao and D. Zhu, "The Application of Ultrasound to the Inspection of Insulation", IEEE $5^{\text {th }}$ Intern. Conf. Conduction and Breakdown in Solid Dielectr., pp. 590-594, 1995.
[7] T. Takada, "Acoustic and Optical Methods for Measuring Electric Charge Distribution in Dielectrics", IEEE Trans. Dielectr. Electr. Insul., Vol. 6, pp. 519-547, 1999.

[8] Y. L. Sam, P. L. Lewin, A. E. Davies, J. S. Wilkinson, S. J. Sutton, and S. G. Swingler, "Surface Discharge Measurements of Polymeric Materials", IEE Proc. Sci. Measur. Techn., Vol. 150 , pp. 43-52, 2003.

[9] A. Zargari and T. R. Blackburn, "Modified Optical Fibre Sensor for PD Detection in High-Voltage Power Equipment", IEEE Intern. Sympos. Electr. Insul., pp. 424-427, Montreal, Canada, 1996.

[10] A. Zargari and T. R. Blackburn, "Acoustic Detection of Partial Discharges using Non-Intrusive Optical Fibre Sensors", IEEE Intern. Conf. Conduction and Breakdown in Solid Dielectr., pp. 573-576, Sweden, 1998.

[11] T. Y. Kim, K. S. Suh, J. H. Nam and T. Takada, "Acoustic Monitoring of HV Equipment with Optical Fiber Sensor", IEEE Trans. Dielectr. Electr. Insul., Vol. 10, pp. 266-270, 2003.

[12] D. Pommerenke, Report on the partial discharge measurements done in Southampton, 2001.

[13] N. Bessant, Remote Partial Discharge Monitoring in XLPE Cabls via Laser Polarisation Modulation, M. Eng. Project report, University of Southampton, 2002.

[14] D. Pommerenke and K. Masterson, "A novel concept for monitoring partial discharge on EHV-cable system accessories using no active component at the accessories", 8th Intern. Conf. Dielectr. Materials, Measurements and Applications, Edinburgh, UK, pp. 145-149, 2000.

[15] J. Wilson and J. Hawkes, "Optoelectronics", Published by Prentice Hall Europe, 1998.

[16] Y. L. Sam, "Surface Charge Measurement By the Pockels Effect," Ph.D. Thesis, University of Southampton, 2001.

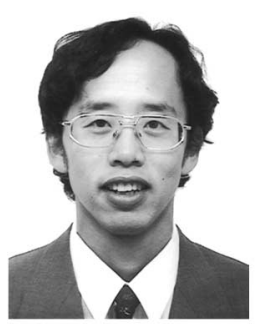

Yuan Tian was born in Liaoning, China in 1970. He received the B.Sc. and M.Sc. degrees in electrical engineering from Xi'an Jiaotong University and Shenyang University of Technology, China, in 1992 and 1995, respectively. He obtained the Ph.D. degree in electrical engineering from the University of Southampton, UK in 2001. Since January 2001 he has been a Research Fellow in electrical power engineering at the University of Southampton. His main research interests include partial discharge measurements, condition monitoring and diagnosis of $\mathrm{HV}$ cables and power plant, non-destructive testing techniques, space charge measurements in dielectrics and applied signal processing techniques. Since 1999 he has published about 20 refereed journal and conference papers in these research areas.

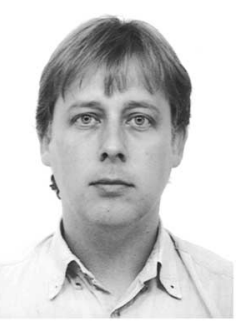

Paul Lewin was born in Ilford, Essex in 1964. He spent a year as a student apprentice at GEC Electrical Projects, Rugby. He received the B.Sc. (Hons) and Ph.D. degrees in electrical engineering from the University of Southampton, UK in 1986 and 1994, respectively. He joined the academic staff of the University in 1989 and at present is a Senior Lecturer in electrical power engineering in the Department of Electronics and Computer Science. His research interests include condition monitoring of $\mathrm{HV}$ cables and plants, surface charge measurement, $\mathrm{HV}$ insulation/dielectric materials and applied signal processing. Since 1996 he has published over 40 refereed conference and journal papers in these research areas. He is currently an Acting Director of the High Voltage Laboratory at the University of Southampton. 


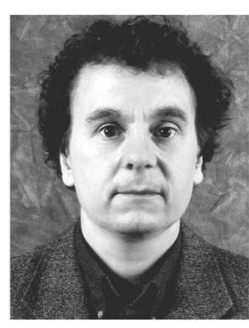

David Pommerenke received a diploma and the Ph.D. degree from the Technical University Berlin in 1989 and 1995, respectively, while he was researching and teaching EMC and High Voltage. In 1996, he joined Hewlett Packard in Roseville, CA as EMC engineer. In 2001, he accepted a position as Associate Professor in the EMC group at the University Missouri-Rolla. His areas of interest are EMC, electrostatic discharge, numerical methods, and partial discharge detection.

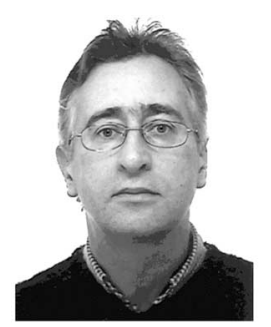

James Wilkinson received the B.Sc. (Eng) degree in electronics in 1977 and the Ph.D. degree in the field of integrated optics in 1985, both from University College London. From 1977 to 1979 he was with the GEC Hirst Research Centre working on optical fibre telecommunications systems. From 1983 to 1985 he was with the Department of Nephrology of St Bartholomew's Hospital, London, working on sensing and control for haemodialysis procedures. He is now Professor of Optoelectronics in the Department of Electronics \& Computer Science, University of Southampton, UK. He is partially seconded to the Optoelectronics Research Centre, where he leads the Integrated Optics and Microstructures Group investigating integrated optical devices including chemical and biochemical sensors with particular application to water quality monitoring, and planar waveguide lasers and amplifiers.

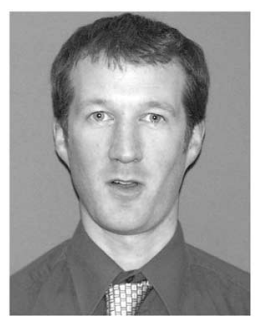

Simon Sutton is the Condition and Capability Team Leader with National Grid Transco and is based at their Head Office in Coventry, UK. Formerly, he was the Cable Policy Team Leader responsible for a wide range of technical issues relating to power cables on the National Grid system including research and development into XLPE cables. $\mathrm{He}$ obtained the B.Sc. and Ph.D. degrees in physics from the University of Reading before holding a Royal Society JSPS Fellowship for 2 years in Japan. During his industrial and academic career he has published over 30 papers on the properties and application of polymeric materials. 